\title{
Intergenerational equity, efficiency, and constructibility
}

\author{
Luc Lauwers \\ Center for Economic Studies, K.U.Leuven, \\ Naamsestraat 69, 3000 Leuven, Belgium; e-mail: Luc.Lauwers@econ.kuleuven.be *
}

July 20, 2010.

\begin{abstract}
.
Global environmental issues - like biodiversity conservation or climate change - are in reality long term issues that are not properly taken into account with traditional models that incorporate the impatience axiom manifested in fixed discount factors and in the use of present discounted utility criteria. When both the short and the very long run are important, one can appeal to overtaking criteria and Chichilnisky criteria. Unfortunately, overtaking criteria are highly incomplete. In order to decrease this incompleteness, stronger anonymity (or equity) axioms were developed. I show that a maximal anonymity axiom compatible with Pareto is a non-constructible object; its existence relies on the Axiom of Choice. The Chichilnisky criterion is based upon two axioms: non dictatorship of the present and non dictatorship of the future. Here, the very long run is captured by a finitely additive measure. Such a measure is a nonconstructible object and has therefore no explicit description.
\end{abstract}

\section{Introduction}

Global environmental issues are long term issues. Traditional discounting is unable to take the long run into account. In contrast, overtaking and Chichilnisky criteria do select long run strategies. The next example recalls these observations. Then, we further discuss $(i)$ the incompleteness of the overtaking criterion, and ( $i i)$ one of the Chichilnisky axioms (non-dictatorship of the present). In particular, we indicate a route to decrease the

*I thank Norbert Brunner, Graciela Chichilnisky, Koen Decancq, Marc Fleurbaey, Johan Quaegebeur, and Luc Van Liedekerke for helpful conversations. A first version was presented at the workshop "Intergenerational equity in climate negotiations, overlapping generations and social welfare" organized by Claude d'Aspremont and Thierry Bréchet (CORE, April 27-28, 2006). This text presents research results of the Belgian Programme of Interuniversity Poles of Attraction initiated under the Science Policy Programmes of the Prime Minister's Office, Belgium. The scientific responsibility is assumed by its authors. 
incompleteness of overtaking, and we show that non-dictatorship of the present involves non-constructible mathematics. We provide a balanced interpretation of these results.

Example. An economy uses trees as a necessary input to production or consumption. The dynamics of tree reproduction are as follows. If $n$ out of $2 n$ subsequent generations cut the forest at a maximal rate, the species become extinct after the $2 n$ 'th generation, in which case there is zero utility at every period from then on. This strategy results in utility streams of the form $u^{n}=(0, \ldots, 0 ; 1, \ldots, 1 ; 0,0, \ldots)$ with the first (resp. last) 1 at the $n+1$ (resp. $2 n$ )'th place, in which generations $n+1, \ldots, 2 n$ cut at a full capacity and exhaust the forest. When the consumption of the forest is delayed and $n$ becomes larger, the forest slightly expands and more generations can benefit. Alternatively, generations can invest in the forest and only cut at an equilibrium rate which allows the forest to survive. This strategy results in the utility stream $u^{\infty}=(.20, .20, \ldots, .20, \ldots)$ in which each generation reaches the same utility level.

We evaluate the different policies by means of the normalized ${ }^{1}$ discounting rule

$$
u=\left(u_{1}, u_{2}, \ldots, u_{t}, \ldots\right) \longmapsto D_{\beta}(u)=(1-\beta)\left(u_{1}+\beta u_{2}+\cdots+\beta^{t-1} u_{t}+\cdots\right) .
$$

We obtain $D_{\beta}\left(u^{n}\right)=\beta^{n}-\beta^{2 n}$ and $D_{\beta}\left(u^{\infty}\right)=.20$. For each $\beta$ in the open interval $(0,1)$, there exists an $n^{*}$ such that $\beta^{n^{*}}-\beta^{2 n^{*}}=.25$. Optimization with respect to a discounting rule leads to the elimination of this forest. ${ }^{2}$ If we judge the long term future important, then we should use the right tools to evaluate a long run policy.

The literature on intergenerational equity provides such tools. ${ }^{3}$ Let me focus on two criteria. First, the overtaking criterion considers an infinite stream $u$ better than $v$ if for some $T$ in $\mathbb{N}_{0}$, the undiscounted sum $u_{1}+u_{2}+\cdots+u_{t}$ is larger than $v_{1}+v_{2}+\cdots+v_{t}$ as soon $t \geq T$. According to this criterium, the sustainable stream $u^{\infty}$ is better than $u^{n}$ for each $n$. Second, Chichilnisky (1996) proposes a convex sum " $C_{\lambda, \beta}=\lambda D_{\beta}+(1-\lambda)$ Lim" of the discounting rule and a value that captures the limiting behavior of the utility stream. Since $\lim _{t \rightarrow \infty} u^{n}=0$ and $\lim _{t \rightarrow \infty} u^{\infty}=.2$, we obtain

$$
C_{\lambda, \beta}\left(u^{\infty}\right)=.2 \lambda+.2(1-\lambda)=.20, \quad \text { and } \quad C_{\lambda, \beta}\left(u^{n^{*}}\right)=.25 \lambda .
$$

As soon as the weight $\lambda$ of the discounting rule is less then .80 , the Chichilnisky criterion $C_{\lambda, \beta}$ ranks the sustainable stream $u^{\infty}$ at the top.

Both criteria have their merits and shortcomings. First, the overtaking criterion combines equity or finite anonymity and Pareto but fails completeness. This is an inevitable consequence of the Lauwers (2010a)-Zame (2007) impossibility result: the existence of a complete, equitable, and Paretian criterion relies on non-constructive mathematics (such as the Axiom of Choice). Recent contributions in this track of literature concentrate on constructible, equitable, and Paretian criteria. For example, the imposition of an

\footnotetext{
${ }^{1}$ An evaluation $F$ of infinite utility streams is said to be normalized if $F(r, r, \ldots, r, \ldots)=r$ for each $r$ in $\mathbb{R}$. Due to this normalization the discounted sum is premultiplied with $(1-\beta)$.

${ }^{2}$ This conclusion extends to, for example, the widely used Dasgupta-Heal-Solow growth model.

${ }^{3}$ Asheim (2010) provides an excellent survey.
} 
anonymity demand stronger than finite anonymity decreases incompleteness; ${ }^{4}$ e.g. Lauwers (1997), Fleurbaey and Michel (2003), Mitra and Basu (2007), Banerjee (2006), Asheim, d'Aspremont, Banerjee (2010), Asheim and Banerjee (2010), and Kagama and Kojima (2009a, b). In this note, I explore the boundaries of combining different equity principles and different Pareto principles without imposing completeness. I show that a maximal equity principle, compatible with Pareto, is a non-constructible object.

Second, Chichilnisky (1996, 2009b) translates the requirement of equal treatment for the present and the future into two new axioms for sustainable development. These two axioms (non dictatorship of the present and non dictatorship of the future $)^{5}$ in combination of independence, characterize the class $C_{\lambda, \beta}$ of sustainable social welfare functions. Unfortunately, in many economic models of growth there does not exist a utility stream that is optimal under this criterion. Recent contributions in this track of literature concentrate on this issue of applicability; e.g. Heal (1998), Li and Löfgren (2000), and Figuières and Tidball (2010). Furthermore, Alvarez-Cuadrado and Long (2009) defend the axioms of non dictatorship, propose a Bentham-Rawls criterion, and show the existence of optimal paths. Similarly, Asheim, Mitra, and Tungodden (2010) introduce the concept of a sustainable recursive social welfare function, of which Asheim and Mitra's (2010) sustainable discounted utilitarianism is special case. Their criteria also satisfy the two Chichilnisky axioms and are applicable. ${ }^{6}$ Besides this problem of applicability, there is a problem of non-constructibility. The "distinct future"-part in the Chichilnisky criterion is an integral against a purely finitely additive measure. Such a measure is a non-constructible object. ${ }^{7}$

The results that 'maximal anonymity' and 'finitely additive measures' involve nonconstructive mathematics should be interpreted with care. The use of non-constructive mathematics within economic theory is well known. For example, Debreu's proof of the second welfare theorem (each Pareto allocation can be realized as the market equilibrium of some economy) uses - similar to the proof of existence of Chichilnisky criterianon-constructive mathematics (see Chichilnisky, 2009a, 2010). In the practical context of optimal growth, however, an explicit description of the ordering describing social prefer-

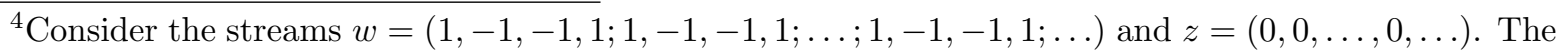
overtaking criterion is unable to rank $w$ and $z$. An utilitarian overtaking criterion that satisfies fixed step anonymity considers $w$ and $z$ equally good.

${ }^{5} \mathrm{~A}$ welfare function displays 'dictatorship of the present' if it is insensitive for changes that affect the distinct future. A welfare function displays 'dictatorship of the future' if it is insensitive for changes that do not affect the limiting behavior criterion.

${ }^{6}$ Burniaux and Martins (2010), Chipman and Martins (2010), Dutta and Radner (2010), Karp and Zhang (2010), and Ostrom (2010) tackle the question of how to implement policies that respect the interests of future generations and to assess their effectiveness in the context of global externalities with long-lasting effects. Lecocq and Hourcade (2010) argue that optimal policies may require estimates of future intragenerational distributions. Rezai, Foley, and Taylor (2010) show that, in some cases, such policies may benefit all generations.

${ }^{7}$ Purely finitely additive measures are typically obtained via non-constructive mathematics (HahnBanach's theorem or ultrafilters, cf. Chichilnisky, 2009a, 2010). This observation can be strengthened: it is impossible to create a purely finitely additive measure on $\mathbb{N}_{0}$ without recurse to non-constructive methods.
} 
ences would generally be needed in order to compute the optimal path (Fleurbaey, Michel, 2003, p794).

In view of these remarks, I propose the following positive interpretation. First, the result on maximal anonymity should be seen as an additional defense of the 'fixed step anonymity' axiom. Fixed step anonymity is stronger than finite anonymity, decreases incomparability, and is based upon the constructible group of fixed step permutations. Second, the Chichilnisky criterion can be made constructible by restricting the domain to, for example, those infinite utility streams which exhibit a well defined and finite limiting behavior (see Chichilnisky, 2009a). In this restricted domain, the limiting value is well defined (without recurse to non-constructive mathematics) and captures the long run value. Also, one can weaken the independence axiom and allow for alternatives to capture the very long run behavior. For example, the maps lim inf and limsup do not involve nonconstructive mathematics, violate independence, ${ }^{8}$ and fit in the Chichilnisky approach.

The next section collects preliminaries. Subsection 2.1 recalls the notions of a social welfare relation and the basic notations. Subsection 2.2 characterizes Pareto-compatible anonymity demands (Mitra and Basu, 2007). Surprisingly, an anonymity demand is compatible with strong Pareto if and only if it is compatible with weak Pareto. Subsection 2.3 recalls the Axiom of Choice and the notion of ultrafilter. Section 3 develops the main result: a maximal anonymity condition involves an ultrafilter on the lattice of partitions. As a consequence, the group of fixed step permutations is not maximal. Section 4 concentrates on the non-constructibility of purely finitely additive measures.

\section{Preliminaries}

\subsection{Social welfare relations}

Let $\mathbb{N}_{0}=\{1,2,3, \ldots\}$ denote the set of positive integers and $\mathbb{R}$ the set of real numbers. Let the interval $Y \subseteq \mathbb{R}$ be the set of all possible utility levels. We assume that $Y$ contains 0 and 1 . The set $X=Y^{\mathbb{N}_{0}}$ collects all possible utility streams and is called the domain. An infinite utility stream $x$ is a vector in $X$. Each $x$ in $X$ can be viewed as a map from $\mathbb{N}_{0}$ to $Y$, associating with each $t$ in $\mathbb{N}_{0}$ the element $x_{t}$ in $Y$. Vector inequalities are denoted $\leq$, $<$, and $\ll$. For each $x$ in $X, \lim \inf (x)$ is the infimum (and $\lim \sup (x)$ is the $\operatorname{supremum}$ ) of the set of accumulation points of $x$.

A social welfare relation (SWR) is a reflexive and transitive binary relation in the domain $X$. The symmetric and the asymmetric component of the SWR $\precsim$ are denoted by $\sim$ and $\prec$. The SWR $\precsim$ is complete if for each $x$ and $y$ in $X$ we have that either $x \precsim y$ or $y \precsim x$. The SWR $\precsim_{1}$ is a subrelation to a SWR $\precsim_{2}$ if for each $x$ and $y$ in $X$ we have $(i)$ $x \precsim_{1} y$ implies $x \precsim_{2} y$ and $\left(\right.$ ii) $x \prec_{1} y$ implies $x \prec_{2} y$.

A permutation $\pi$ on $\mathbb{N}_{0}$ is a one-to-one map from $\mathbb{N}_{0}$ to $\mathbb{N}_{0}$. For each $x$ in $X$, the composite map $x \circ \pi$ is a map from $\mathbb{N}_{0}$ to $Y$ and can be written as the infinite utility

\footnotetext{
${ }^{8}$ The map lim inf (see Section 2.1) is not additive: $\lim \inf (1,0,1,0, \ldots)=\lim \inf (0,1,0,1, \ldots)=0$; while $\lim \inf [(1,0,1,0, \ldots)+(0,1,0,1, \ldots)]=\liminf (1,1,1,1, \ldots)=1$.
} 
stream

$$
x \circ \pi=\left(x_{\pi(1)}, x_{\pi(2)}, \ldots, x_{\pi(t)}, \ldots\right) .
$$

Let $\operatorname{Sym}\left(\mathbb{N}_{0}\right)$ collect all permutations on $\mathbb{N}_{0}$. The set $\operatorname{Sym}\left(\mathbb{N}_{0}\right)$ when equipped with the composition operation becomes a group. The next definition collects a monotonicity demand, two infinite versions of the Pareto axiom, and one concept related to permutations.

\section{Definition.}

- A SWR $\precsim$ is monotonic if for each $x$ and $y$ in $X$ we have that $x \leq y$ implies $x \precsim y$.

- A SWR $\precsim$ satisfies the Pareto axiom if $\precsim$ is monotonic and for each $x$ and $y$ in $X$ we have that $x<y$ implies $x \prec y$.

- A SWR $\precsim$ satisfies the weak Pareto axiom if $\precsim$ is monotonic and for each $x$ and $y$ in $X$, we have that $x \ll y$ implies $x \prec y$.

- Let $\mathcal{Q}$ be a group of permutations. A SWR $\precsim$ satisfies $\mathcal{Q}$-anonymity if for each $\pi$ in $\mathcal{Q}$ and for each $x$ in $X$ we have $x \sim x \circ \pi$.

The Pareto axiom, also known as the strong Pareto axiom, postulates sensitivity in each coordinate. The SWRs represented by the maps liminf and limsup combine completeness, $\operatorname{Sym}\left(\mathbb{N}_{0}\right)$-anonymity, and monotonicity, and violate weak Pareto. Indeed, the infinite sequences $z=(0,0, \ldots, 0, \ldots)$ and $y=(1,1 / 2, \ldots, 1 / k, \ldots)$ have one single accumulation point $(\lim z=\lim y=0)$, have the same liminf- and limsup-values, and satisfy $z \ll y$. Chambers (2009) characterizes both SWRs.

With respect to anonymity, we only consider groups of permutations that include the group of finite permutations. Hereby, the permutation $\pi$ is said to be finite if there exists a $T$ in $\mathbb{N}_{0}$ such that $\pi(t)=t$ for each $t \geq T$. Let $\mathcal{Q}_{\text {fn }}$ collect all finite permutations. A SWR is said to be finite anonymous if it satisfies $\mathcal{Q}_{\mathrm{fn}}$-anonymity. The overtaking criterion satisfies finite anonymity, the Chichilnisky criterion violates finite anonymity. Furthermore, a permutation $\pi$ is said to be fixed step if there exists a natural number $n$, such that $\pi(\{1,2, \ldots, k n\})=\{1,2, \ldots, k n\}$ for each $k$ in $\mathbb{N}_{0}$. Let $\mathcal{Q}_{\mathrm{fs}}$ collect all fixed step permutations. Observe the inclusion $\mathcal{Q}_{\mathrm{fn}} \subset \mathcal{Q}_{\mathrm{fs}}$. Finally, for each group $\mathcal{Q}$ of permutations we define the SWR $\precsim_{\mathcal{Q}}$ as follows: for each $x$ and $y$ in $X$, we have

$$
x \precsim_{\mathcal{Q}} y \quad \text { if and only if there is a } \pi \text { in } \mathcal{Q} \text { such that } x \circ \pi \leq y \text {. }
$$

This relation is $\mathcal{Q}$-anonymous, reflexive (the identity permutation belongs to the group $\mathcal{Q}$ ), and transitive (the group $\mathcal{Q}$ is closed under composition).

\subsection{Pareto-compatible permutations}

Anonymity axioms are based upon groups of permutations. In this subsection we characterize permutations that are compatible with Pareto. 
Let $\pi$ be a permutation on the set $\mathbb{N}_{0}$. The vector $\left(k, \pi(k), \pi^{2}(k), \pi^{3}(k), \ldots\right)$ is said to be the cycle generated by $\pi$ on $k$ in $\mathbb{N}_{0}$. Each permutation can be written as a succession of cycles on disjoint sets (Hall, 1976, Chapter 5). For example, the permutation

$$
\pi_{1}=(1,2)(3,4)(5,6) \cdots(2 n-1,2 n) \cdots
$$

switches the odd and even numbers: for each $n$ in $\mathbb{N}_{0}$ the number $2 n-1$ is mapped upon $2 n$ and $2 n$ is mapped upon $2 n-1$. The final element in a cycle is mapped upon the first element in that cycle. The permutation

$$
\pi_{2}=(1)(2,3)(4,5) \cdots(2 n, 2 n+1) \cdots
$$

keeps the number 1 fixed and then switches the even and odd numbers. A permutation on $\mathbb{N}_{0}$ might generate a cycle of infinite length. The permutation

$$
\pi_{3}=(\ldots, 9,7,5,3,1,2,4,6,8, \ldots)
$$

maps 1 upon 2. Furthermore, $\pi_{3}$ maps an even number upon its even successor and an odd number upon its odd predecessor, as such $\pi_{3}(123)=121$ and $\pi_{3}(100)=102$. We keep the references $\pi_{1}, \pi_{2}$, and $\pi_{3}$ throughout this note. The decomposition of a permutation into pairwise disjoint cycles is unique, except for the order in which the cycles are written, also within each cycle the numbers are allowed to be permuted cyclically. As such, the permutations $(1,2)(3)(4,5,6,7)$ and $(3)(1,2)(5,6,7,4)$ coincide.

A permutation representable by an infinite sequence of finite cycles is said to be cyclic. The permutations $\pi_{1}$ and $\pi_{2}$ are cyclic, $\pi_{3}$ is not cyclic. Finite permutations and fixed step permutations are cyclic. The set of all cyclic permutations is denoted by $\mathcal{P}$. The set $\mathcal{P}$ is not a group: the composition $\pi_{1} \circ \pi_{2}$ of two cyclic permutations results in the permutation $\pi_{3}$. The next lemma highlights the main motivation to study cyclic permutations. The lemma already appeared in Mitra and Basu (2007, Lemma 1). Their proof uses coordinatewise convergent sequences of infinite utility streams. The proof below only uses 0-1-utility streams and therefore strengthens their result.

Lemma 1. A permutation $\pi$ is cyclic if and only if there is no $x$ in $X$ satisfying $x<x \circ \pi$. Proof. The only-if-part is straightforward. If the permutation $\pi$ is cyclic, then it can be decomposed as an infinite juxta position of permutations on finite sets. Each permutation on a finite set is unable to conflict with the Pareto principle.

The if-part (if there is no conflict with Pareto, then the permutation is cyclic) is done by contraposition. Hence, consider a permutation $\pi$ with an infinite cycle at $m$ in $\mathbb{N}_{0}$ :

$$
\left(\ldots, \pi^{-4}(m), \pi^{-3}(m), \pi^{-2}(m), \pi^{-1}(m), m, \pi^{1}(m), \pi^{2}(m), \pi^{3}(m), \pi^{4}(m), \ldots\right) .
$$

Relabel this cycle (let 1 denote $m$ ) to obtain the cycle $\pi_{3}$ and consider the following table:

$$
\begin{aligned}
& \pi_{3}=(\ldots, \quad 9, \quad 7, \quad 5, \quad 3, \quad 1, \quad 2, \quad 4, \quad 6, \quad 8, \ldots), \\
& x=(\ldots, \quad 0, \quad 0, \quad 0, \quad 0, \quad 0, \quad 1, \quad 1, \quad 1, \quad 1, \quad \ldots), \\
& y=x \circ \pi_{3}=(\ldots, \quad 0, \quad 0, \quad 0, \quad 0, \quad 1, \quad 1, \quad 1, \quad 1, \quad 1, \ldots) \text {. }
\end{aligned}
$$


The first line in this table is a cycle of infinite length. The second line presents an infinitely long utility stream in $X$. This utility stream is made up of two sequences, a sequence of 'ones' is attached to the even positions $\left(x_{2 n}=1\right)$ and a sequence of zeros is attached to the odd positions $\left(x_{2 n-1}=0\right)$. The final line presents the permuted utility stream $y=x \circ \pi_{3}$ (recall that $\left.y_{i}=x_{\pi(i)}\right)$. The utility stream $y$ dominates $x$ (indeed, $x_{1}<y_{1}$ ).

The infinite cycle $\pi_{3}$ generates a second domination result. There exists an $x$ in $X$ such that $x \ll\left(x \circ \pi_{3}\right)$ :

$$
\begin{aligned}
& \pi_{3}=(\ldots, \quad 9, \quad 7, \quad 5, \quad 3, \quad 1, \quad 2, \quad 4, \quad 6, \quad 8, \quad \ldots) \text {, } \\
& x=\left(\ldots, \quad \frac{1}{9}, \quad \frac{1}{7}, \quad \frac{1}{5}, \quad \frac{1}{3}, \quad 1, \quad 2-\frac{1}{2}, \quad 2-\frac{1}{4}, \quad 2-\frac{1}{6}, \quad 2-\frac{1}{8}, \ldots\right), \\
& x \circ \pi_{3}=\left(\ldots, \quad \frac{1}{7}, \quad \frac{1}{5}, \quad \frac{1}{3}, \quad 1, \quad 2-\frac{1}{2}, \quad 2-\frac{1}{4}, \quad 2-\frac{1}{6}, \quad 2-\frac{1}{8}, \quad 2-\frac{1}{10}, \ldots\right) \text {. }
\end{aligned}
$$

Lemma 1, thus, holds when Pareto is weakened to weak Pareto. We summarize. Let $\mathcal{Q}$ be a group of permutations. Then,

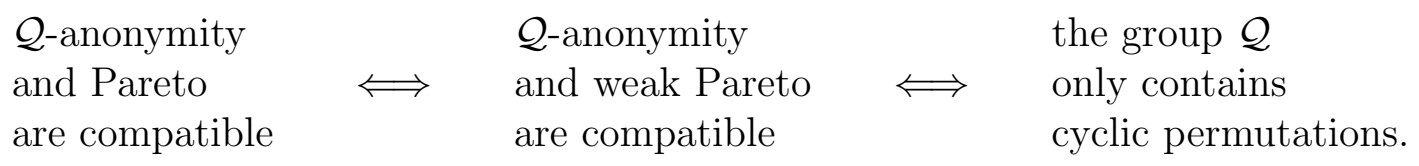

Within the class of transitive and reflexive relations, there is no trade-off between the Pareto axioms and anonymity. Finally, if $\mathcal{Q}$ is a group of cyclic permutations, then $(i)$ the relation $\precsim_{\mathcal{Q}}$ extends the Suppes-Sen grading principle, $(i i)$ is the smallest (for inclusion) SWR that satisfies $\mathcal{Q}$-anonymity and Pareto (Banerjee, 2006), and (iii) satisfies

- $x \sim_{\mathcal{Q}} y$ if and only if there exists a $\pi$ in $\mathcal{Q}$ such that $x \circ \pi=y$, and

- $x \prec_{\mathcal{Q}} y$ if and only if there exists a $\pi$ in $\mathcal{Q}$ such that $x \circ \pi<y$.

We only verify the first item. Suppose both $x \precsim_{\mathcal{Q}} y$ and $y \precsim_{\mathcal{Q}} x$ hold. Then there exist two permutations $\pi$ and $\sigma$ in $\mathcal{Q}$ such that $x \circ \pi \leq y$ and $y \circ \sigma \leq x$. Therefore,

$$
x \circ \pi \circ \sigma \leq y \circ \sigma \leq x .
$$

Since $\mathcal{Q}$ is a group, the permutation $\pi \circ \sigma$ is cyclic. The inequalities become equalities, hence $y \circ \sigma=x$, and $x \sim_{\mathcal{Q}} y$.

\subsection{The Axiom of Choice, ultrafilters}

The Axiom of Choice $(\mathrm{AC})$ postulates for each nonempty family $\mathcal{D}$ of nonempty sets the existence of a function $f$ such that $f(S) \in S$ for each set $S$ in the family $\mathcal{D}$. The function $f$ is referred to as a choice function. AC does not provide an explicit way to construct such a choice function and provoked considerable criticism in the aftermath of Zermelo's 
formulation in $1904 .{ }^{9}$ AC implies a number of paradoxes such as the decomposition of a sphere into a sphere of smaller size and the existence of a nonmeasurable set of real numbers. The nonconstructive character of AC is further revealed by Dianonescu (1975) who showed that AC implies the law of the excluded middle. ${ }^{10}$ Constructive mathematics rejects the law of the excluded middle and hence rejects $\mathrm{AC}$.

This note appeals to the non-constructible object "free ultrafilter". We will use free ultrafilters $(i)$ in the definition of maximal groups of cyclic permutations (Section 3), and (ii) in the definition of the "distinct future"-part of the Chichilnisky criterion (Section 4). The definition of a filter and an ultrafilter is as follows.

Let $S$ be a set. A filter on $S$ is a nonempty family $\mathcal{F}$ of subsets of $S$ that satisfies

- $\varnothing$ is not in $\mathcal{F}$,

- if $A$ and $B$ are in $\mathcal{F}$, then $A \cap B$ is in $\mathcal{F}$ (intersection property),

- if $A$ is in $\mathcal{F}$ and $A \subseteq B$, then $B$ is in $\mathcal{F}$.

If, in addition,

- for each $A \subseteq S$, either $A \in \mathcal{F}$ or $S-A \in \mathcal{F}$,

then $\mathcal{F}$ is an ultrafilter. An ultrafilter is a filter that is maximal for inclusion. The family of all subsets of $S$ that contain a given element $s$ of $S$ is an ultrafilter on $S$ and is said to be principal. An ultrafilter $\mathcal{F}$ that is not principal is said to be free and satisfies $\cap_{\mathcal{F}} A=\varnothing$. AC (reformulated as Zorn's lemma) implies the existence of free ultrafilters on infinite sets. The non-constructiveness of free ultrafilters is well known (Jech, 1973).

\section{Maximal Pareto-compatible anonymity conditions}

From Subsection 2.2 we know that Pareto-compatible anonymity axioms are based upon groups of 'cyclic' permutations. This section introduces partition groups of cyclic permutations. We show that a maximal (for inclusion) partition group of cyclic permutations involves an ultrafilter on the lattice of partitions and is therefore a non constructible object.

The notion of a filter on sets extends to a filter on a lattice of partitions. Let us recall the definitions (Halbeisen and Löwe, 2001). A partition of $\mathbb{N}_{0}$ is a family of pairwise disjoint nonempty sets such that their union coincides with $\mathbb{N}_{0}$. If $A$ and $B$ are two partitions of $\mathbb{N}_{0}$, we say that $A$ is coarser than $B$ (or that $B$ is finer than $A$ ) and we write $A \sqsubseteq B$ if each piece in $A$ is a union of pieces of $B$. The coarsest partition of $\mathbb{N}_{0}$ (everything in one

\footnotetext{
${ }^{9} \mathrm{AC}$ is $(i)$ consistent and $(i i)$ independent: $(i) \mathrm{AC}$ can be added to the Zermelo-Fraenkel axioms of set theory (ZF) without yielding a contradiction, and (ii) AC is not a theorem of ZF (Fraenkel et al, 1973).

${ }^{10}$ The law of the excluded middle states the truth of ' $P$ or not- $P$ ' for each proposition $P$ and can be used to claim the existence of certain objects without any hint to its construction. For example, the real number $c=\sqrt{2}^{\sqrt{2}}$ either is rational (in which case one sets $a=b=\sqrt{2}$ ) or is not rational (in which case one sets $a=c$ and $b=\sqrt{2}$ ). Conclude the existence of irrational numbers $a$ and $b$ for which $a^{b}$ is rational.
} 
piece) is denoted by $0=\left\{\mathbb{N}_{0}\right\}$, the finest partition (all pieces of which are singletons) by 1. Each partition is in between 0 and 1 .

Let $\Omega_{0}$ collect those partitions of $\mathbb{N}_{0}$ that consist out of infinitely many finite pieces. Partitions containing one (or more) infinite piece(s) are not distinguished, they are denoted by 0 . We endow the class $\Omega=\Omega_{0} \cup\{0\}$ with two operations $\cup$ and $\cap$. The partition $A \cup B$ is the coarsest partition in $\Omega$ that refines $A$ and $B$, and the partition $A \cap B$ is the finest partition in $\Omega$ that is coarser than $A$ and $B$. In case the partition $A \cap B$ contains an infinite piece, we put $A \cap B$ equal to 0 . The couple $(\Omega, \sqsubseteq)$ is a lattice.

A filter on the lattice $(\Omega, \sqsubseteq)$ is a collection $\mathcal{F}$ of members of $\Omega$ that satisfies

- 0 is not in $\mathcal{F}$,

- if both $A$ and $B$ are in $\mathcal{F}$, then $A \cap B$ is in $\mathcal{F}$,

- if $B$ is in $\mathcal{F}$ and $B \sqsubseteq A$ (with $A$ in $\Omega$ ), then $A$ is in $\mathcal{F}$.

A family $\mathcal{B} \subseteq \Omega$ is said to be a filter base if $(i) 0 \notin \mathcal{B}$, and $(i i)$ for each $A_{1}$ and $A_{2}$ in $\mathcal{B}$, there is a $B$ in $\mathcal{B}$ such that $B \sqsubseteq A_{1} \cap A_{2}$. In case $\mathcal{B}$ is a filter base, then the family

$$
\mathcal{B}^{+}=\{A \in \Omega \mid \text { there is a } B \text { in } \mathcal{B} \text { such that } B \sqsubseteq A\}
$$

is a filter on the lattice $(\Omega, \sqsubseteq)$. The filter $\mathcal{B}^{+}$coincides with the intersection of all filters that include $\mathcal{B}$. A filter that is maximal for inclusion is said to be an ultrafilter. Each ultrafilter $\mathcal{F}$ on $(\Omega, \sqsubseteq)$ is free, i.e. $\bigcap\{A \mid A \in \mathcal{F}\}=0$.

We recall two facts on ultrafilters (Facts 2.1-2 in Halbeisen and Löwe, 2001, p321).

- A family $\mathcal{F}$ is an ultrafilter on $(\Omega, \sqsubseteq)$ if and only if for each $A$ in $\Omega$ either $A \in \mathcal{F}$ or there is a $B$ in $\mathcal{F}$ such that $A \cap B=0$ (the 'either-or' being exclusive).

- If $\mathcal{B}$ is a family of elements of $\Omega$ with the finite intersection property (for each finite subfamily $\left\{A_{1}, A_{2}, \ldots, A_{n}\right\} \subseteq \mathcal{B}$ we have $\left.A_{1} \cap A_{2} \cap \ldots \cap A_{n} \neq 0\right)$, then there is an ultrafilter $\mathcal{F}$ on $(\Omega, \sqsubseteq)$ with $\mathcal{B} \subseteq \mathcal{F}$.

The second fact is implied by AC (reformulated as Zorn's lemma). The notion "ultrafilter on a lattice" generalizes the notion "free ultrafilter on a set". The next example clarifies this statement.

Example. Each infinite subset $S=\left\{n_{1}, n_{2}, \ldots, n_{k}, \ldots\right\}$, with $n_{1}<n_{2}<\cdots<n_{k}<\cdots$, of $\mathbb{N}_{0}$ induces a partition

$$
V_{S}=\left\{\left\{1,2, \ldots, n_{1}\right\},\left\{n_{1}+1, n_{1}+2, \ldots, n_{2}\right\}, \ldots,\left\{n_{k}+1, n_{k}+2, \ldots, n_{k+1}\right\}, \ldots\right\} .
$$

Now, let $\mathcal{F}_{\mathbb{N}_{0}}$ be a free filter on the set $\mathbb{N}_{0}$. Then, the family of all partitions generated by elements in $\mathcal{F}_{\mathbb{N}_{0}}$, denoted by $\mathcal{F}_{\Omega}=\left\{V_{S} \in \Omega \mid S \in \mathcal{F}_{\mathbb{N}_{0}}\right\}$, is a filter on the lattice $(\Omega$, $)$. Moreover, $\mathcal{F}_{\Omega}$ is an ultrafilter on the lattice $(\Omega, \sqsubseteq)$ if and only if $\mathcal{F}_{\mathbb{N}_{0}}$ is a free ultrafilter on the set $\mathbb{N}_{0}$. 
The link towards cyclic permutations is as follows. Each permutation partitions the set $\mathbb{N}_{0}$ : present the permutation as a juxta position of cycles and replace the brackets ( and ) by $\{$ and $\}$. Each cyclic permutation partitions the set $\mathbb{N}_{0}$ into an infinite sequence of finite sets. For example, the partition induced by the permutation $\pi_{1}$ is equal to

$$
\operatorname{Part}\left(\pi_{1}\right)=\{\{1,2\},\{3,4\}, \ldots,\{2 n-1,2 n\}, \ldots\} .
$$

Consider the partition $A=\left\{N_{1}, N_{2}, \ldots, N_{k}, \ldots\right\}$ in $\Omega_{0}$. We will refer to

$$
\operatorname{Sym}(A)=\operatorname{Sym}\left(N_{1}\right) \times \operatorname{Sym}\left(N_{2}\right) \times \cdots \times \operatorname{Sym}\left(N_{k}\right) \times \cdots,
$$

with $\operatorname{Sym}\left(N_{k}\right)$ the group of all permutations on the finite set $N_{k}$, as the symmetric group of the partition $A$. The group $\operatorname{Sym}(A)$ stabilizes the partition $A$, i.e. this group collects all the permutations with an induced partition that is equal to or finer than $A$. We shorten $\operatorname{Sym}(\operatorname{Part}(\pi))$ to $\operatorname{Sym}(\pi)$. A group $\mathcal{Q}$ of permutations that includes $\operatorname{Sym}(\pi)$ for each $\pi$ in $\mathcal{Q}$ is said to be a partition group.

An anonymity condition based upon a partition group $\mathcal{Q}$ of cyclic permutations is Pareto-compatible. In order to enlarge the group $\mathcal{Q}$ (towards a maximal subgroup of cyclic permutations), we add a cyclic permutation to the group $\mathcal{Q}$, and we consider the group generated by $\mathcal{Q}$ and this additional permutation. The next lemma investigates the effect of enlarging a partition group, its proof is in the Appendix.

Lemma 2. Let $\sigma_{A}$ and $\sigma_{B}$ be two cyclic permutations on $\mathbb{N}_{0}$. Then, $\operatorname{Sym}\left(\sigma_{B}\right)$ contains a permutation $\rho$ such that $\rho \circ \sigma_{A}$ generates the partition $\operatorname{Part}\left(\sigma_{A}\right) \cap \operatorname{Part}\left(\sigma_{B}\right)$.

Adding a cyclic permutation to a partition group smuggles in permutations with courser partitions. As such, one runs the risk of ending up with a non-cyclic permutation. E.g. the addition of $\pi_{1}$ to the partition group $\operatorname{Sym}\left(\pi_{2}\right)$ generates the non-cyclic permutation $\pi_{3}$.

We continue with some further notation. Let $\mathcal{B}$ be a family of partitions in $\Omega$. Consider the set

$$
\{\pi \mid \text { there is a } B \text { in } \mathcal{B} \text { such that } B \sqsubseteq \operatorname{Part}(\pi)\}
$$

of all permutations that stabilize an element of $\mathcal{B}$. Denote by $\mathcal{Q}_{\mathcal{B}}$ the smallest partition group that includes this set of stabilizers. If $\mathcal{B}$ is a filter base, then $\mathcal{Q}_{\mathcal{B}}$ and $\mathcal{Q}_{\mathcal{B}^{+}}$coincide. For example, let FS collect the partitions

$$
\{\{1,2, \ldots, n\},\{n+1, n+2, \ldots, 2 n\}, \ldots,\{k n+1, k n+2, \ldots,(k+1) n\}, \ldots\}
$$

with $n=1,2, \ldots$. The family $F S$ is a filter base and the partition group $\mathcal{Q}_{F S}$ coincides with the group $\mathcal{Q}_{\mathrm{fs}}$ of fixed step permutations. Proposition 1 characterizes maximal partition groups.

Proposition 1. Let $\mathcal{B}$ be a family of partitions in $\Omega$. Then, $\mathcal{Q}_{\mathcal{B}}$ is a maximal group of cyclic permutations if and only if $\mathcal{B}^{+}$is an ultrafilter. 
Proof. The if-part. Let $\mathcal{B}^{+}$be a filter. Then, $0 \notin \mathcal{B}$, and $\mathcal{Q}_{\mathcal{B}}$ only contains cyclic permutations. If $\pi$ and $\rho$ belong to $\mathcal{Q}_{\mathcal{B}}$, then $\operatorname{Part}(\pi) \cap \operatorname{Part}(\rho)$ belongs to $\mathcal{B}^{+}$. Hence, $\mathcal{Q}_{\mathcal{B}}$ is closed for composition. Next, observe that the partition induced by a permutation coincides with the partition induced by its inverse permutation. Therefore, $\mathcal{Q}_{\mathcal{B}}$ is a (partition) group of cyclic permutations.

Now, suppose that $\mathcal{B}^{+}$is an ultrafilter. We have to show that $\mathcal{Q}_{\mathcal{B}}$ is maximal. Therefore, assume that the cyclic permutation $\pi$ is not in $\mathcal{Q}_{\mathcal{B}}$. The induced partition $A=\operatorname{Part}(\pi)$ does not belong to the ultrafilter $\mathcal{B}^{+}$. Hence, there is a $B$ in $\mathcal{B}^{+}$such that $A \cap B=0$. Lemma 2 implies the existence of a permutation in $\operatorname{Sym}(B)$ such that the composition with $\pi$ induces the partition 0 . This composed permutation has an infinite cycle. Therefore, the permutation $\pi$ cannot be added to $\mathcal{Q}_{\mathcal{F}}$ to generate a larger group of cyclic permutations.

The only-if-part. Let $\mathcal{Q}_{\mathcal{B}}$ be a maximal subgroup of cyclic permutations. We have to show that $\mathcal{B}^{+}$is an ultrafilter. Since only cyclic permutations are involved, $0 \notin \mathcal{B}$. Next, assume that the partition $A$ is not in $\mathcal{B}^{+}$. We have to show the existence of a partition $B$ in $\mathcal{B}^{+}$with $A \cap B=0$. A permutation $\pi$ that induces $A$ does not belong to $\mathcal{Q}_{\mathcal{B}}$. Since the group $\mathcal{Q}_{\mathcal{B}}$ is maximal, there is a $\sigma$ in $\mathcal{Q}_{\mathcal{B}}$ such that $\pi \circ \sigma$ is not cyclic. Conclude that $A \cap \operatorname{Part}(\sigma) \sqsubseteq \operatorname{Part}(\pi \circ \sigma)=0$ with $\operatorname{Part}(\sigma)$ in $\mathcal{B}^{+}$.

Mitra and Basu (2007) formulate the question whether the group $\mathcal{Q}_{\mathrm{fs}}$ of fixed step permutations is a maximal (for inclusion) group of cyclic permutations. Proposition 1 answers this question in the negative. The filter generated by the family $F S$ is not an ultrafilter and the partition group $\mathcal{Q}_{F S}=\mathcal{Q}_{\mathrm{fs}}$ is not maximal.

There is one further concern. We should check whether larger partition groups (and stronger anonymity demands) reduce the incompleteness of the social welfare relation. Let the partition group $\mathcal{G}^{\prime}$ be larger than the partition group $\mathcal{G}$. Then, the relation $\precsim_{\mathcal{G}}$ is a subrelation to $\precsim_{\mathcal{G}^{\prime}}$. The next proposition studies the indifference sets of these relations and uses the concept of permissible permutations. The definition is as follows. Let $\precsim$ be a SWR in $X$. The set of permissible partitions is defined as

$$
\Pi(\precsim)=\{A \in \Omega \mid \text { for each } \pi \text { in } \operatorname{Sym}(A) \text { and for each } x \text { in } X \text { we have } \pi(x) \sim x\} .
$$

If the SWR $\precsim_{1}$ is a subrelation to the Paretian SWR $\precsim_{2}$, then $\Pi\left(\precsim_{1}\right) \subseteq \Pi\left(\precsim_{2}\right)$. If, in addition $\Pi\left(\precsim_{1}\right)$ is a strict subset of $\Pi\left(\precsim_{2}\right)$, then $\precsim_{1}$ is a strict subrelation to $\precsim_{2}$ (i.e. $\precsim_{2}$ is less incomplete than $\precsim_{1}$ ). Proposition 2 investigates the link between partition groups and permissible partitions. We use $\precsim_{\mathcal{B}}$ as a shorthand for the social welfare relation $\precsim_{\mathcal{Q}_{\mathcal{B}}}$.

Proposition 2. Let the family $\mathcal{B}$ of partitions in $\Omega$ be a filter base. Then, the relation $\precsim_{\mathcal{B}}$ is reflexive, transitive, Paretian, and $\mathcal{B}$-anonymous. Furthermore, the set $\Pi\left(\precsim_{\mathcal{B}}\right)$ of permissible partitions coincides with the filter $\mathcal{B}^{+}$.

Proof. The conditions imposed upon $\mathcal{B}$ turn $\mathcal{Q}_{\mathcal{B}}$ into a partition group of cyclic permutations. This group $\mathcal{Q}_{\mathcal{B}}$ coincides with $\mathcal{Q}_{\mathcal{B}^{+}}$. Mitra and Basu (2007, Proposition 3) show that for each group $\mathcal{G}$ of cyclic permutations, the relation $\precsim_{\mathcal{G}}$ is reflexive, transitive, Paretian, and $\mathcal{G}$-anonymous. Apply their result for $\mathcal{G}=\mathcal{Q}_{\mathcal{B}}$ and conclude that $\precsim_{\mathcal{B}}$ satisfies the properties as listed. 
Let us now verify that $\Pi\left(\precsim_{\mathcal{B}}\right)$ coincides with $\mathcal{B}^{+}$. The inclusion $\mathcal{B}^{+} \subseteq \Pi\left(\precsim_{\mathcal{B}}\right)$ is immediate. In case $\mathcal{B}^{+}$is an ultrafilter also the reverse inclusion holds (otherwise, there exists a cyclic permutation $\pi$ outside the group $\mathcal{Q}_{\mathcal{B}}$ that keeps the indifference relation; as $\mathcal{Q}_{\mathcal{B}}$ is maximal $\mathcal{Q}_{\mathcal{B}} \cup\{\pi\}$ generates noncyclic permutations and a contradiction is obtained).

There remains one single statement to be proved: the inclusion $\Pi\left(\precsim_{\mathcal{B}}\right) \subseteq \mathcal{B}^{+}$under the assumption that $\mathcal{B}^{+}$is not an ultrafilter. We show this inclusion by contradiction and assume $A \notin \mathcal{B}^{+}$. There exists an ultrafilter $\mathcal{F}$ that extends $\mathcal{B}$ and does not contain $A$ (in the family $\mathcal{A}$ of all filters which do not contain $A$ each chain has a maximal element, so by Zorn's lemma $\mathcal{A}$ has a maximal element that appears to be an ultrafilter; cf. Ax, 1968, Section 11a). The relation $\precsim_{\mathcal{B}}$ is a subrelation to $\precsim_{\mathcal{F}}$, and $A \notin \Pi\left(\precsim_{\mathcal{F}}\right)$. Hence, $A$ does not belong to $\Pi\left(\precsim_{\mathcal{B}}\right)$.

Propositions 1 and 2 justify the statements claimed in the introduction. Anonymity demands are formulated in terms of groups of cyclic permutations. We focussed on partition groups of cyclic permutations. Enlarging the partition group, strengthens the anonymity demand, and decreases the incomparability. The strongest Pareto-compatible anonymity demand based upon a partition group of cyclic permutations, involves an ultrafilter on the lattice of partitions and is therefore a non-constructible object.

\section{Measures on $\mathbb{N}_{0}$, the Chichilnisky criterion}

A finitely additive measure $\mu$ on $\mathbb{N}_{0}$ assigns to each subset of $\mathbb{N}_{0}$ a nonnegative real number and assigns to the union of two disjoint sets the sum of their numbers. The measure $\mu$ is said to be countably additive if the measure of a countable union of pairwise disjoint sets is equal to the sum of the measures of those sets. The finitely additive measure $\nu$ is dominated by $\mu$ (and we write $\nu \leq \mu$ ) is for each subset $S$ of $\mathbb{N}_{0}$, we have $\nu(S) \leq \mu(S)$. The finitely additive measure $\mu$ is said to be purely finitely additive if the inequalities $0 \leq \nu \leq \mu$ with $\nu$ countably additive imply that $\nu=0$. From Yosida and Hewitt (1952) and Rao (1958) we know that each finitely additive measure uniquely decomposes as the sum of a countably additive and a purely additive measure. This decomposition result is at the heart of the Chichilnisky criterion: the discounting rule (non dictatorship of the future) takes the role of the countably additive measure and the "distinct future"-part (non dictatorship of the present) is a purely finitely additive measure.

Typically, a purely finitely additive measure is obtained by means of Hahn-Banach's theorem or by means of a free ultrafilter (e.g. Chichilnisky, 2009a, 2009b). We only describe the second route.

A free ultrafilter $\mathcal{F}$ on $\mathbb{N}_{0}$ defines a limit on $X$. Consider a sequence $x$ in $X$ and all of its limit points. Each limit point is the limit of a subsequence. There is only one limit point with a converging subsequence $x_{i_{1}}, x_{i_{2}}, \ldots, x_{i_{t}}, \ldots$ for which the set $\left\{i_{1}, i_{2}, \ldots, i_{t}, \ldots\right\}$ of indices belongs to $\mathcal{F}$. Define $\lim _{\mathcal{F}}(x)=\lim _{t \rightarrow \infty} x_{i_{+}}$. Due to the intersection property of $\mathcal{F}$, we have $\lim _{\mathcal{F}}(x+y)=\lim _{\mathcal{F}}(x)+\lim _{\mathcal{F}}(y)$ for each $x$ and $y$ in $X$. The ultrafilter-based-limit 
$\lim _{\mathcal{F}}$ defines a finitely additive measure:

$$
\mu_{\mathcal{F}}(S)=\lim _{\mathcal{F}} s_{t} \quad \text { with } s_{t}=\frac{\#(S \cap\{1,2, \ldots, t\})}{t},
$$

and $S$ a subset of $\mathbb{N}_{0}$. If the sequence $s_{1}, s_{2}, \ldots, s_{t}, \ldots$ has only one accumulation point, then $\mu_{\mathcal{F}}(S)$ coincides with 'the' limit of this sequence and is known as the natural density of $S$. For example, the set of even numbers has a natural density equal to .5; the set of all multiples of 20 has a natural density equal to .05. Unfortunately, not every subset of $\mathbb{N}_{0}$ has a natural density. For example, the set

$$
S_{1}=\{1,10,11, \ldots, 19,100,101, \ldots, 199,1000,1001, \ldots\}
$$

of all natural numbers having their first digit equal to 1 has no natural density. The measure $\mu_{\mathcal{F}}\left(S_{1}\right)$ depends upon the particular (non-constructible) ultrafilter $\mathcal{F}$ and can take any value between $1 / 9$ and $5 / 9 .{ }^{11}$

Both routes to obtain purely finitely additive measures (Hahn-Banach's theorem and a free ultrafilter) rely upon AC. As a consequence, both ways to obtain a purely finitely additive measure involve non-constructive methods. Obviously, one cannot conclude from this that purely finitely additive measures are non-constructible objects. The knowledge that non-constructive methods can be used to obtain a purely finitely additive measure, does not answer the question whether a purely finitely additive measure can be obtained without recurse to non-constructive methods.

The question whether or not a purely finitely additive measure on $\mathbb{N}_{0}$ is a constructible object is tackled by Lauwers (2010b). Not surprisingly, the answer is negative: the existence of a purely finitely measure relies upon AC.

Proposition 3 (Lauwers, 2010b). The existence of a purely finitely additive measure on $\mathbb{N}_{0}$ entails the existence of a non-Ramsey set (from Mathias (1977) we know that a non-Ramsey set is a non-constructible object).

As mentioned in the introduction, the question of how to evaluate policies that involve the distant future is normative and should by no means be answered through the Axiom of Choice. Only constructible and well defined criteria can take part in the discussions. Although the maps lim inf and limsup violate additivity (cf. footnote 8), they provide a constructible way to capture the 'distinct future'-part of an infinite path. A convex combination of a discounting rule, lim inf, and lim sup remains in the spirit of the Chichilnisky criteria. Furthermore, Chichilnisky criteria are constructible when applied to a restricted domain, e.g. the domain of infinite paths which have a well defined and finite limiting behavior. In such a restricted domain, 'the' limit of a path is defined, captures the distinct future value, and does not depend upon non-constructive methods.

\footnotetext{
${ }^{11}$ In this example, $\lim \inf \left(s_{t}\right)$ is the limit of the sequence $1 / 9,11 / 99,111 / 999, \ldots$ and is equal to $1 / 9$; $\lim \sup \left(s_{t}\right)$ is the limit of the sequence $1,11 / 19,111 / 199, \ldots$ and is equal to $5 / 9$.
} 


\section{Appendix, proof of Lemma 2}

Lemma 2 connects the partition induced by the product of two cyclic permutations to the intersection of the partitions induced by the permutations. In general, the relation $\operatorname{Part}\left(\sigma_{1}\right) \cap \operatorname{Part}\left(\sigma_{2}\right) \sqsubseteq \operatorname{Part}\left(\sigma_{1} \circ \sigma_{2}\right)$ holds. For example, consider the following cyclic permutations:

$$
\begin{aligned}
& \sigma_{1}=(1)(2,3,5,6,7,4)(8,11,13,14,15,12,10,9) \underline{(16,19,21,22,23,20,18,17)} \cdots \\
& \sigma_{2}=(1,2,3)(4,8,10,11,7,5)(6)(9)(12,16,18,19,15,13)(14)(17) \underline{(20,24,26,27,23,21)(22)(25)} \cdots .
\end{aligned}
$$

The representation continues by repeating the underlined cycles taking into account a shift of +8 . Here, $\operatorname{Part}\left(\sigma_{1}\right) \cap \operatorname{Part}\left(\sigma_{2}\right)=\mathbb{N}_{0}$ while both compositions $\sigma_{2} \circ \sigma_{1}$ and $\sigma_{1} \circ \sigma_{2}$ are cyclic:

$$
\begin{aligned}
& \sigma_{2} \circ \sigma_{1}=\pi_{1}=(1,2)(3,4)(5,6)(7,8) \cdots, \text { and } \\
& \sigma_{1} \circ \sigma_{2}=(1,3)(2,5)(4,11)(6,7)(8,9)(10,13)(12,19)(14,15)(16,17)(18,21) \cdots
\end{aligned}
$$

Lemma 2. Let $\sigma_{A}$ and $\sigma_{B}$ be two cyclic permutations on $\mathbb{N}_{0}$. Then, $\operatorname{Sym}\left(\sigma_{B}\right)$ contains a permutation $\rho$ such that $\rho \circ \sigma_{A}$ generates the partition $\operatorname{Part}\left(\sigma_{A}\right) \cap \operatorname{Part}\left(\sigma_{B}\right)$.

Proof. Denote $A=\operatorname{Part}\left(\sigma_{A}\right)$ and $B=\operatorname{Part}\left(\sigma_{B}\right)$. We prove the lemma in case $C=A \cap B$ consists out of an infinite number of finite sets. In case the partition $C$ contains an infinite piece, the same ideas apply.

Without loss (otherwise re-enumerate $\mathbb{N}_{0}$ ), we assume the existence of an increasing sequence $n_{1}, n_{2}, \ldots$, $n_{k}, \ldots$ in $\mathbb{N}_{0}$ such that the partition $C$ can be written as

$$
C=\{\underbrace{\left[1, n_{1}\right]}_{S},\left[n_{1}+1, n_{2}\right], \ldots,\left[n_{k}+1, n_{k+1}\right], \ldots\} .
$$

Both $A$ and $B$ are finer than $C$. We focus on one of the pieces in $C$, say $S=\left[1, n_{1}\right]$. Again, without loss, we assume that the restriction of $\sigma_{A}$ to $S$ is as follows

$$
\left.\sigma_{A}\right|_{S}=\left(1,2, \ldots, k_{1}\right)\left(k_{1}+1, k_{1}+2, \ldots, k_{2}\right) \cdots\left(k_{m-1}+1, k_{m-1}+2, \ldots, n_{1}\right) .
$$

Denote the partition classes by $S_{1}=\left[1, k_{1}\right], S_{2}=\left[k_{1}+1, k_{2}\right], \ldots, S_{m}=\left[k_{m-1}+1, n_{1}\right]$.

We construct a permutation $\rho$ in $\operatorname{Sym}\left(\left.B\right|_{S}\right)$ by induction. The partition $A \cap B$ - when restricted to $S$ is equal to $S$. Hence, there exists a couple $\left(\ell_{1}, \ell^{1}\right)$ in $S_{1} \times\left(S-S_{1}\right)$ both belonging to one piece of $B$. Put $\rho\left(\ell_{1}\right)=\left(\ell^{1}\right)$. Let $\ell^{1}$ belong to $S^{1}=S_{i}$. Move on to the set $S_{2}=S_{1} \cup S^{1}$. Again, there exists a couple $\left(\ell_{2}, \ell^{2}\right)$ in $S_{2} \times\left(S-S_{2}\right)$ that both belong to one piece of $B$. Put $\rho\left(\ell_{2}\right)=\ell^{2}$. This procedure ends after $m$ steps. Put the permutation $\rho$ equal to $\left(\ell_{1}, \ell^{1}\right)\left(\ell_{2}, \ell^{2}\right) \cdots\left(\ell_{m}, \ell^{m}\right)$, elements of $S$ that are not listed remain fixed.

The permutation $\rho \circ \sigma_{A}$ generates the cycle $S$ in one piece. Repeat the whole construction for the other pieces in $C$ and paste together the corresponding permutations to obtain the result.

\section{References}

Alvarez-Cuadrado F, Van Long N (2009) A mixed Bentham-Rawls criterion for intergenerational equity: theory and implications. Journal of Environmental Economics and Management 58, 154-168.

Asheim GB (2010) Intergenerational equity. Annual Review of Economics 2 (2010) 197-222.

Asheim GB, d'Aspremont C, Banerjee K (2010) Generalized time-invariant overtaking. Journal of Mathematical Economics (forthcoming, doi: 10.1016/j.jmateco.2010.03.005).

Asheim GB, Banerjee K (2010) Fixed-step anonymous overtaking and catching up. International Journal of Economic Theory 6, 149-165. 
Asheim GB, Mitra T (2010) Sustainability and discounted utilitarianism in models of economic growth. Mathematical Social Sciences, 59, 148-169.

Asheim GB, Mitra T, Tungodden B (2010) Sustainable recursive social welfare functions. Economic Theory (this issue).

Ax J (1968) The elementary theory of finite fields. Annals of Mathematics 88, 239-271.

Banerjee K (2006) On the extension of the utilitarian and Suppes-Sen social welfare relations to infinite utility streams. Social Choice and Welfare 27, 327-339.

Burniaux J-M, Martins JO (2010) Carbon leakages: a general equilibrium view. Economic Theory (this issue).

Chambers CP (2009) Intergenerational equity: sup, inf, limsup, and liminf. Social Choice and Welfare $32(2), 243-252$.

Chichilnisky G (1996) An axiomatic approach to sustainable development. Social Choice and Welfare $13,231-257$.

Chichilnisky G (2009a) The topology of fear. Journal of Mathematical Economics 45, 807-816.

Chichilnisky G (2009b) Avoiding extinction: equal treatment of the present and the future. Economics: The Open-Access, Open-Assessment E-Journal 3, 2009-32.

http://www.economics-ejournal.org/economics/journalarticles/2009-32

Chichilnisky (2010) Sustainable markets with short sales. Economic Theory (this issue).

Chipman JS, Tian G (2010) Detrimental externalities, pollution rights, and the Coase theorem. Economic Theory (this issue).

Dianonescu R (1975) Axiom of choice and complementation. Proceedings of the American Mathematical Society 51(1), 176-178.

Dutta PK, Radner R (2010) Capital growth in a global warming model: will China and India sign a climate treaty? Economic Theory (this issue).

Figuières C, Tidball M (2010) Sustainable exploitation of a natural resource: A satisfying use of Chichilnisky's criterion. Economic Theory (this issue).

Fleurbaey M, Michel P (2003) Intertemporal equity and the extension of the Ramsey criterion. Journal of Mathematical Economics 39, 777-802.

Halbeisen L, Löwe B (2001) Ultrafilter spaces on the semilattice of partitions. Topology and its Applications $115,317-332$.

Hall M Jr (1976) The theory of groups. Chelsea Publishing Company, New York.

Heal GM (1998) Valuing the future: Economic theory and sustainability. New York: Columbia University Press.

Jech TJ (1973) The axiom of choice. North-Holland.

Kamaga K, Kojima T (2009a) $\mathcal{Q}$-anonymous social welfare relations on infinite utility streams. Social Choice and Welfare 33(3), 405-413.

Kamaga K, Kojima T (2009b) On the leximin and utilitarian criteria with extended anonymity. Waseda University, Tokyo, Japan.

Karp L, Zhang J (2010) Taxes versus quantities for a stock pollutant with endogenous abatement costs and asymmetric information. Economic Theory (this issue). 
Lauwers L (1997) Infinite utility: insisting on strong monotonicity. Australasian Journal of Philosophy $75(2), 222-233$.

Lauwers L (2010a) Ordering infinite utility streams comes at the cost of a non-Ramsey set. Journal of Mathematical Economics 46, 32-37.

Lauwers L (2010b) Purely finitely additive measures are non-constructible objects. DPS Center for Economic Studies, K.U.Leuven. http://www.econ.kuleuven.be/ces/discussionpapers/Dps10/Dps1010.pdf

Lecocq F, Hourcade J-C (2010) Unspoken ethical issues in the climate affair insights from a theoretical analysis of negotiation mandates. Economic Theory (this issue).

Li C-Z, Löfgren K-G (2000) Renewable resources and economic sustainability: a dynamic analysis with heterogeneous time preferences. Journal of Environmental Economics and Management 40, 236-250.

Mathias ARD (1977) Happy families. Annals of Pure and Applied Logic 12, 59-111.

Mitra T, Basu K (2007) On the existence of Paretian social welfare relations for infinite utility streams with extended anonymity. In Roemer J, Suzumura K (eds.), Intergenerational Equity and Sustainability, Palgrave, London, 85-99.

Moore GH (1982) Zermelo's axiom of choice: its origins, development, and influence. New York.

Ostrom E (2010) Nested externalities and polycentric institutions: must we wait for global solutions to climate change before taking actions at other scales? Economic Theory (this issue).

Rao RR (1958) A note on finitely additive measures. The Indian Journal of Statistics 19, 27-28.

Rezai A, Foley DK, Taylor L (2010) Global warming and economic externalities. Economic Theory (this issue).

Yosida K, Hewitt E (1952) Finitely additive measures. Transactions of the American Mathematical Society $72,46-66$.

Zame W (2007) Can intergenerational equity be operationalized? Theoretical Economics 2, 187-202. 